\title{
FAR-INFRARED SOURCE COUNTS AT 70 AND 160 MICRONS IN SPITZER DEEP SURVEYS
}

\author{
H. Dole, ${ }^{1,2}$ E. Le Floc’h, ${ }^{1}$ P. G. Pérez-González, ${ }^{1}$ C. Papovich, ${ }^{1}$ E. Egami, ${ }^{1}$ G. Lagache, ${ }^{2}$ \\ A. Alonso-Herrero, ${ }^{1}$ C. W. Engelbracht,${ }^{1}$ K. D. Gordon, ${ }^{1}$ D. C. Hines, ${ }^{1,3}$ O. Krause, ${ }^{1}$ K. A. Misselt, ${ }^{1}$ \\ J. E. Morrison, ${ }^{1}$ G. H. Rieke, ${ }^{1}$ M. J. Rieke, ${ }^{1}$ J. R. Rigby, ${ }^{1}$ E. T. Young, ${ }^{1}$ L. Bai, ${ }^{1}$ M. Blaylock, ${ }^{1}$ \\ G. Neugebauer, ${ }^{1}$ C. A. Beichman, ${ }^{4}$ D. T. Frayer, ${ }^{5}$ J. R. Mould ${ }^{6}{ }^{\text {and P. L. Richards }}{ }^{7}$ \\ Received 2004 March 26; accepted 2004 May 6
}

\begin{abstract}
We derive galaxy source counts at 70 and $160 \mu \mathrm{m}$ using the Multiband Imaging Photometer for Spitzer (MIPS) to map the Chandra Deep Field-South (CDF-S) and other fields. At $70 \mu \mathrm{m}$, our observations extend upward about 2 orders of magnitude in flux density from a threshold of $15 \mathrm{mJy}$, and at $160 \mu \mathrm{m}$ they extend about an order of magnitude upward from $50 \mathrm{mJy}$. The counts are consistent with previous observations on the bright end. Significant evolution is detected at the faint end of the counts in both bands, by factors of 2-3 over no-evolution models. This evolution agrees well with models that indicate that most of the faint galaxies lie at redshifts between 0.7 and 0.9 . The new Spitzer data already resolve about 23\% of the cosmic far-infrared background at $70 \mu \mathrm{m}$ and about $7 \%$ at $160 \mu \mathrm{m}$.
\end{abstract}

Subject headings: galaxies: evolution — galaxies: statistics — infrared: galaxies

\section{INTRODUCTION}

The cosmic infrared background (CIB), relic emission of the formation and evolution of the galaxies, peaks in the farinfrared (FIR) in the $60-200 \mu \mathrm{m}$ wavelength range (Puget et al. 1996; Hauser et al. 1998; Lagache et al. 1999; Gispert et al. 2000; Hauser \& Dwek 2001). In the local universe only about a third of the extragalactic emission is released in the FIR (Soifer \& Neugebauer 1991). However, the CIB FIR peak accounts for more than half of the total optical/infrared background, indicating strong evolution of galaxy properties toward high FIR output in the past. Characterizing the galaxies responsible for most of the CIB is therefore an important goal of cosmological surveys. Galaxy counts (or number counts) provide a powerful tool to investigate the evolution of the galaxies and their contribution to the CIB.

The cryogenic infrared space missions of the Infrared Astronomical Satellite (IRAS) and Infrared Space Observatory (ISO) (see Genzel \& Cesarsky 2000 and Dole 2003 for reviews) provided important data on source counts at $60 \mu \mathrm{m}$ (Hacking \& Soifer 1991) and at $100 \mu \mathrm{m}$ (Rowan-Robinson et al. 1986), and more recently at $90 \mu \mathrm{m}$ (Kawara et al. 1998, 2004; Efstathiou et al. 2000; Juvela et al. 2000; Linden-Vornle et al. 2000; Matsuhara et al. 2000; Rodighiero et al. 2003) and $170 \mu \mathrm{m}$ (Kawara et al. 1998, 2004; Puget et al. 1999; Matsuhara et al. 2000; Dole et al. 2001). Mid-infrared (MIR) observation with ISOCAM at $15 \mu \mathrm{m}$ (Elbaz et al. 1999) are also of great interest, since they are believed to resolve a

\footnotetext{
1 Steward Observatory, University of Arizona, 933 North Cherry Avenue, Tucson, AZ 85721.

${ }^{2}$ Institut d'Astrophysique Spatiale, bât 121, Université Paris-Sud, F-91405 Orsay Cedex, France.

3 Space Science Institute, 4750 Walnut Street, Suite 205, Boulder, CO 80301.

4 Michelson Science Center, California Institute of Technology, Pasadena, CA 91125.

5 Spitzer Science Center, California Institute of Technology, Pasadena, CA 91125.

${ }^{6}$ NOAO, 950 North Cherry Avenue, Tucson, AZ, 85719.

7 Department of Physics, 345 Birge Hall, University of California, Berkeley, CA 94720.
}

significant fraction of the CIB into sources (Elbaz 2002). The Spitzer Space Telescope (Werner et al. 2004) provides the ability for much deeper and wider area surveys from 3.6 to $160 \mu \mathrm{m}$. This paper investigates source counts at 70 and $160 \mu \mathrm{m}$ from Spitzer. A companion paper addresses the MIR source counts at $24 \mu \mathrm{m}$ (Papovich et al. 2004). The three-band source counts are the basis of new phenomenological models by Lagache et al. (2004).

\section{OBSERVATIONS AND DATA REDUCTION}

Observations were carried out with the Multiband Imaging Photometer for Spitzer (Rieke et al. 2004) in the Chandra Deep Field-South (CDF-S) and the Boötes field corresponding to the NOAO Deep Wide Field Survey (NDWFS; Jannuzi \& Dey 1999); we also used an engineering MIPS observation of the Marano field. The observational mode (scan map) provides multiple sightings of each source, typically 10 and 60 at $70 \mu \mathrm{m}$ in the Boötes and CDF-S, respectively. However, at $160 \mu \mathrm{m}$, the number of sightings is only typically 2 in Boötes and 12 in CDF-S. See Table 1 and Papovich et al. (2004) for details.

The data were reduced with the Data Analysis Tool (Gordon et al. 2004), from the raw data (ramps) to the final coadded mosaics. The illumination corrections were derived from the data themselves. At $70 \mu \mathrm{m}$, the data have been median-filtered in the time domain before mosaicking. Note that data from $\mathrm{Ge}$ : Ga detectors are always challenging to process; but with MIPS, most of the difficulties are overcome with frequent calibrations (stimulator flashes), which track the responsivity variations. Nevertheless, the noise properties at faint fluxes are still being investigated at both 70 and $160 \mu \mathrm{m}$. In this work, we adopt conservative detection limits. A future paper will address extracting the ultimate sensitivity from these data. Sample images in the CDF-S are shown in Figure 1 (Plate 1).

\section{PHOTOMETRY AND CATALOGS}

To control the sample and the selection function, we accepted source detections only where the redundancy was high (typically $80 \%$ or more of the mean weight), avoiding the 
TABLE 1

LOG OF OBSERVATIONS

\begin{tabular}{|c|c|c|c|c|c|c|c|}
\hline \multirow[b]{2}{*}{$\begin{array}{c}\text { FIELD } \\
\text { NAME }^{a}\end{array}$} & \multirow[b]{2}{*}{$\begin{array}{l}\text { MIPS } \\
\text { AOT }^{b}\end{array}$} & \multicolumn{3}{|c|}{$70 \mu \mathrm{m}$} & \multicolumn{3}{|c|}{$160 \mu \mathrm{m}$} \\
\hline & & $\begin{array}{c}\text { Area } \\
\left(\operatorname{deg}^{2}\right)\end{array}$ & $\begin{array}{l}t_{\text {int }}{ }^{\mathrm{c}} \\
(\mathrm{s})\end{array}$ & $\begin{array}{l}S_{\text {cut }}^{\mathrm{d}} \\
(\mathrm{mJy})\end{array}$ & $\begin{array}{c}\text { Area } \\
\left(\operatorname{deg}^{2}\right)\end{array}$ & $\begin{array}{l}t_{\text {int }}{ }^{\mathrm{c}} \\
(\mathrm{s})\end{array}$ & $\begin{array}{l}S_{\text {cut }}^{\mathrm{d}} \\
\text { (mJy) }\end{array}$ \\
\hline Boötes ....... & Med. & 8.75 & 40 & 80 & 7.70 & 8 & $\ldots{ }^{\mathrm{e}}$ \\
\hline Marano ...... & Slow & 0.42 & 100 & 25 & 0.31 & 20 & 50 \\
\hline CDF-S ....... & Slow & 0.67 & 600 & 15 & 0.54 & 120 & 50 \\
\hline
\end{tabular}

a See Papovich et al. (2004) for details on fields.

b Scan map mode.

c Per sky pixel.

d Flux density at which catalog was cut.

e Field data not used at $160 \mu \mathrm{m}$.

edges and the noisiest areas of the images. The resulting positions were fed to DAOPHOT (Stetson 1987) in IRAF $^{8}$ for PSF fitting. We checked that the residual maps were indeed free of sources.

At $70 \mu \mathrm{m}$ the photometric calibration is derived from many observation campaigns, and its uncertainty is conservatively estimated at the order of $20 \%$. We use only detections at $15 \mathrm{mJy}$ and brighter in the CDF-S, $25 \mathrm{mJy}$ and brighter in Marano, and $80 \mathrm{mJy}$ and brighter in the Boötes field. These flux levels are determined using the sharp decrease in the counts due to the incompleteness effect. At $160 \mu \mathrm{m}$, the calibration is based on a combination of observations of standard stars, asteroids, and comparisons with measurements with other FIR missions (ISO, COBE, and modeling including IRAS measurements). It is also estimated to be accurate to about $20 \%$. We have included in our counts only objects of $50 \mathrm{mJy}$ and brighter in the CDF-S and Marano (levels determined using incompleteness as at $70 \mu \mathrm{m}$ ). Because of the low redundancy level of the $160 \mu \mathrm{m}$ data in the Boötes field, we postpone using it for a later paper.

Catalogs were produced separately at each wavelength; surveys at each wavelength are thus unbiased. Detected FIR sources in the CDF-S sort as follows. For sources selected at $70 \mu \mathrm{m}, 92 \%$ have a $24 \mu \mathrm{m}$ ID; $54 \%$ have a $160 \mu \mathrm{m}$ ID (same with 24 and 160). For sources selected at $160 \mu \mathrm{m}, 98 \%$ have a $24 \mu \mathrm{m}$ ID; 43\% have a $70 \mu \mathrm{m}$ ID (same with 24 and 70).

\section{SOURCE COUNTS}

At $70 \mu \mathrm{m}, 131$ sources were detected down to $15 \mathrm{mJy}$ in the CDF-S, 55 sources down to $25 \mathrm{mJy}$ in Marano, and 117 down to $80 \mathrm{mJy}$ in the Boötes. At $160 \mu \mathrm{m}$, down to $50 \mathrm{mJy}, 123$ sources were detected in the CDF-S, and 89 sources in Marano. The source density corresponds to about 150 beams per source at $70 \mu \mathrm{m}$ and 15 at $160 \mu \mathrm{m}$, using the definition of Helou \& Beichman (1990).

Source counts are given in integral form (Figs. $2 a$ and $3 a$ ) and differential form, divided by the Euclidean component (Figs. $2 b$ and $3 b$ ) at 70 and $160 \mu \mathrm{m}$, respectively.

Note that we did not correct for incompleteness. Error bars on counts are $1 \sigma$ Poisson uncertainty. Bins with less than four sources have not been displayed for clarity, since their

\footnotetext{
${ }^{8}$ IRAF is distributed by the National Optical Astronomy Observatories, which are operated by the Association of Universities for Research in Astronomy, Inc., under the cooperative agreement with the National Science Foundation.
}

significance is low, with uncertainties of $50 \%$ or higher. In addition, photometric uncertainty have been displayed at high flux only. In order to visualize the contribution from each field, source counts have not been merged and have been overplotted. One should keep in mind that MIPS source counts will eventually go deeper and will be corrected for incompleteness.

The observed fields nicely complement each other in terms of area and depth. This allows us to probe a flux range of almost 2 orders of magnitude at $70 \mu \mathrm{m}$. One order of magnitude is covered at $160 \mu \mathrm{m}$. It is possible to check consistency and the cosmic variance in the common flux density range. At $70 \mu \mathrm{m}$, in the range 25 to $100 \mathrm{mJy}$ where three fields overlap in flux density, the differential counts are almost consistent within the error bars. At $160 \mu \mathrm{m}$, in the range 100 to $300 \mathrm{mJy}$, the differential counts are consistent within the error bars. At both wavelengths, number counts in CDF-S appear consistently lower than in Marano.

\section{DISCUSSION}

\section{1. $70 \mu \mathrm{m}$}

The MIPS $70 \mu \mathrm{m}$ counts show a great consistency with the IRAS $60 \mu \mathrm{m}$ counts of Lonsdale et al. (1990) converted at $70 \mu \mathrm{m}$ using $\nu_{60} S_{60}=\nu_{70} S_{70}$.

A selection of recent models is shown in Figure 2, including a nonevolution scenario. The most striking result is the strong excess of MIPS $70 \mu \mathrm{m}$ sources compared to the nonevolution model, a factor of 3 at around 20-30 mJy. Strong evolution had been reported previously at 60 and $90 \mu \mathrm{m}$, and these data provide unambiguous confirmation.

Two models lie close to the data: King \& Rowan-Robinson (2003) and Lagache et al. $(2003,2004)$. These models, developed to fit observables mostly from IRAS, ISO, and SCUBA surveys as well as the CIB spectral energy distribution (SED), are based on a strong evolution of luminous (and ultraluminous) infrared galaxies (LIRGs and ULIRGs, respectively). The latter model predicts a peak in the redshift distributions of resolved sources at $70 \mu \mathrm{m}$ near $z \sim 0.7$ (Dole et al. 2003). Figure $4 a$ shows the galaxy contribution to the differential counts, as a function of redshift, from the Lagache et al. (2004) model. Between $\sim 5$ and $\sim 100 \mathrm{mJy}$, sources at $0.7 \leq$ $z \leq 0.9$ contribute the most to the counts. At brighter fluxes (reached by IRAS and ISO), contributions from local galaxies are more important.

The source counts integrated at $70 \mu \mathrm{m}$ correspond to a brightness of $0.022 \mathrm{MJy} \mathrm{sr}^{-1}$ or $0.95 \mathrm{nW} \mathrm{m}{ }^{-2} \mathrm{sr}^{-1}$. The value of the CIB at this wavelength is not known accurately owing to 


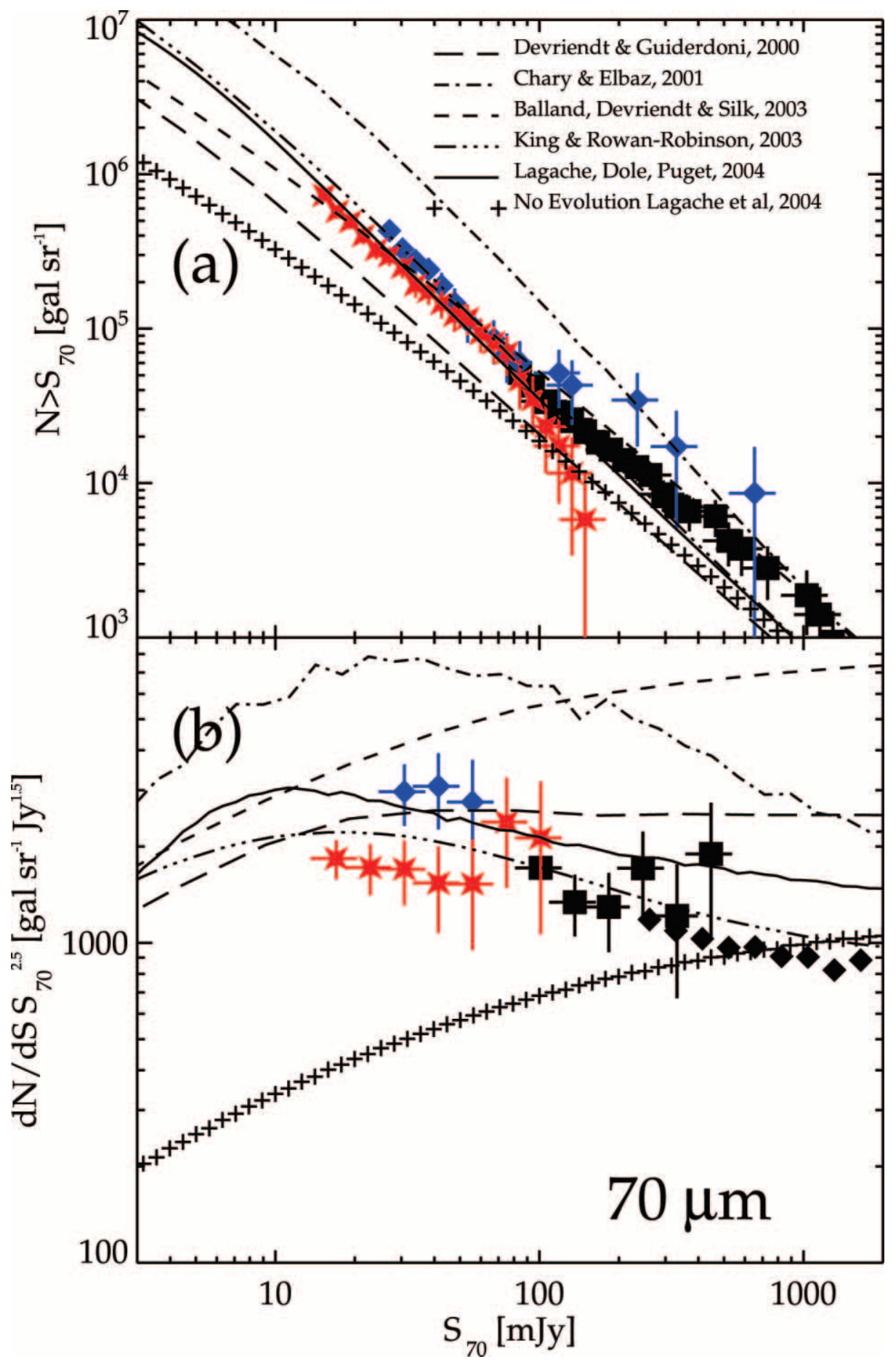

FIG. 2.- Source Counts at $70 \mu \mathrm{m}$ with no correction for incompleteness. Red star, CDF-S; blue diamond, Marano; black square, Boötes Field; black diamond, IRAS $60 \mu \mathrm{m}$ counts from Lonsdale et al. (1990) converted at $70 \mu \mathrm{m}$. (a) Integral source counts. For clarity, photometric uncertainty is only shown for $S_{70 \mu \mathrm{m}}>$ 100 mJy. (b) Differential source counts. Models are also plotted: long dashed line, Devriendt \& Guiderdoni (2000); dash-dotted line, Chary \& Elbaz (2001); dashed line, Balland et al. (2003); dash-dot-dot-dotted line, King \& Rowan-Robinson (2003); solid line, Lagache et al. (2004); plus signs, no-evolution model.

contamination by zodiacal light. If we use the CIB value from the model of Lagache et al. (2004), the MIPS counts show that at $70 \mu \mathrm{m}$ about $23 \%$ of the $70 \mu \mathrm{m} \mathrm{CIB}$ is already resolved.

\section{2. $160 \mu \mathrm{m}$}

At $160 \mu \mathrm{m}$, the comparison with the ISO FIRBACK $170 \mu \mathrm{m}$ survey (Dole et al. 2001) shows that the counts are consistent and within the error bars in the whole common range, 180-
300 mJy. Other ISO observations (Matsuhara et al. 2000; Kawara et al. 2004) agree as well.

Figure 3 overplots the same models as at $70 \mu \mathrm{m}$. The evolution detected at $170 \mu \mathrm{m}$ is confirmed at $160 \mu \mathrm{m}$, down to fainter levels. At about $100 \mathrm{mJy}$, an excess of sources by more than a factor of 2 is observed compared to a nonevolution scenario. Interestingly, as for $70 \mu \mathrm{m}$, the observed evolution is better fitted by the models of Lagache et al. (2004) and King 


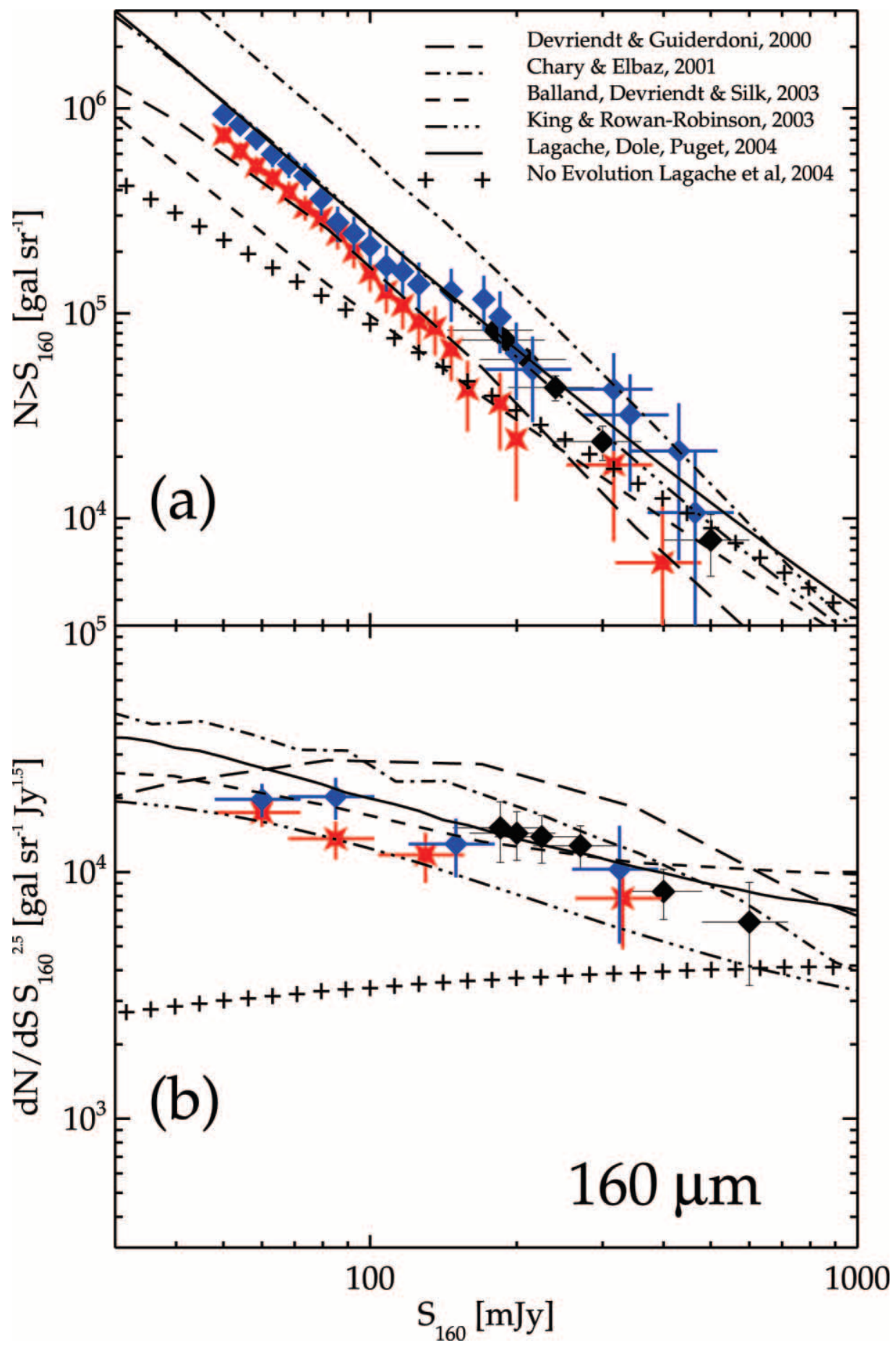

FIG. 3. - Source counts at $160 \mu \mathrm{m}$ with no correction for incompleteness. Red star, CDF-S; blue diamond, Marano field; black diamond, ISO FIRBACK $170 \mu \mathrm{m}$ counts from Dole et al. (2001). (a) Integral source counts. For clarity, photometric uncertainty is only shown for $S_{160 \mu \mathrm{m}}>200 \mathrm{mJy}$. (b) Differential source counts. Models are also plotted with the same symbols as Fig. 2.

\& Rowan-Robinson (2003). The observed slope also agrees with Mould (2003).

We have constrained the bright end of galaxy number counts at $170 \mu \mathrm{m}$ by using data from the ISOPHOT Serendipity Survey (ISOSS). ISOSS provides a total sky coverage of $15 \%$ and is virtually complete at a flux density level of $S_{170 \mu \mathrm{m}}=50 \mathrm{Jy}$. Based on all optically identified galaxies detected by ISOSS (Krause 2003; Stickel et al. 2004) we have derived an integral number density of $n\left(S_{170 \mu \mathrm{m}}>50 \mathrm{Jy}\right)=$
$14 \pm 3$ gal sr $^{-1}$ galaxies at high Galactic latitudes. This point is perfectly matched by the model of Lagache et al. (2003, 2004).

The most striking result of the models that fit the number counts is the existence of two regimes in flux density. In the ISO range (fluxes above $200 \mathrm{mJy}$ ) most of the sources contributing to the counts are local; this is confirmed by observation (Patris et al. 2003). At fainter fluxes, between $\sim 10$ and $200 \mathrm{mJy}$, the counts should be dominated by a population located at redshifts between 0.7 and 0.9 . 


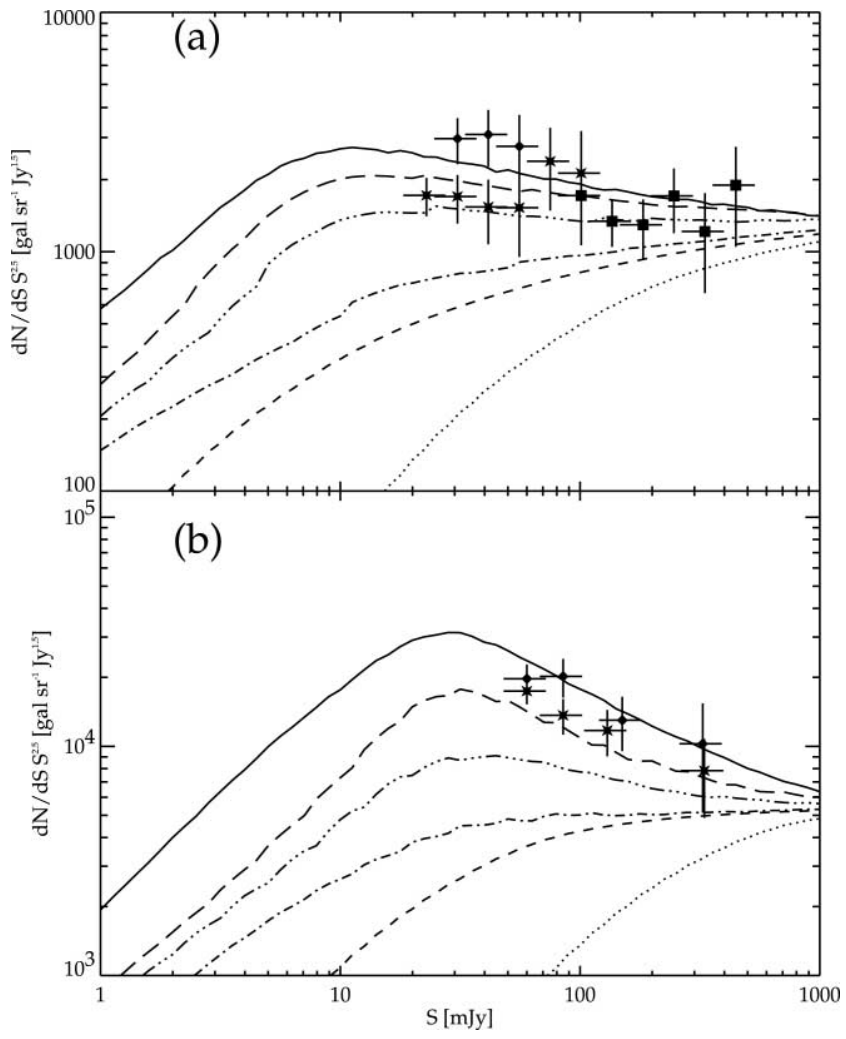

FIG. 4.-Cumulative contributions to the differential source counts at 70 and $160 \mu \mathrm{m}$ of galaxies, as a function of redshift, form the model of Lagache et al. (2004): (a) $70 \mu \mathrm{m}$; (b) $160 \mu \mathrm{m}$. Symbols are described in Figs. 2 and 3. Galaxies contributing to the counts at redshifts 0.1 (dotted line), 0.3 (dashed line), 0.7 (dot-dashed line), 0.9 (triple-dot-dashed line), and 1.1 (long-dashed line). The total contribution is the upper solid line.
The source counts integrated at $160 \mu \mathrm{m}$ correspond to a brightness of $0.07 \mathrm{MJy} \mathrm{sr}^{-1}$ or $1.4 \mathrm{nW} \mathrm{m} \mathrm{m}^{-2} \mathrm{sr}^{-1}$. The CIB value at this wavelength is $1 \mathrm{MJy} \mathrm{sr}^{-1}$ (Lagache et al. 2000); the MIPS counts show that at $160 \mu \mathrm{m}$ about $7 \%$ of the CIB is resolved. Since these counts are preliminary, are not corrected for incompleteness, and are subject to cosmic variance, we anticipate that the actual value might be higher.

\subsection{Concluding Remarks}

The first MIPS far-infrared source counts, spanning about 2 orders of magnitude in flux density at $70 \mu \mathrm{m}$ (and one at $160 \mu \mathrm{m})$, are consistent with previous observations on the bright end and show unambiguous evolution on the faint end. Models predict that most of the sources lie at $z \sim 0.7$ with a tail up to $z \sim 2$ (Dole et al. 2003; Lagache et al. 2003, 2004). This work and companion papers on source counts at $24 \mu \mathrm{m}$ (Papovich et al. 2004), confusion at 24, 70, and $160 \mu \mathrm{m}$ (Dole et al. 2004), and on the interpretation of these new data from the Spitzer cosmological surveys (Lagache et al. 2004) shed new light on the statistical properties of galaxies in an unexplored regime in flux density, and likely in a critical region of redshift space (up to redshifts $z \sim 2$ ) in the FIR (Egami et al. 2004; Le Floc'h et al. 2004).

This work is based on observations made with the Spitzer Space Telescope, which is operated by the Jet Propulsion Laboratory, California Institute of Technology under NASA contract 1407. Support for this work was provided by NASA through contract 960785 issued by JPL/Caltech. We warmly thank J. Cadien and J.-L. Puget, and the IRS Team for providing us with the Boötes data.
Balland, C., Devriendt, J. E. G., \& Silk, J. 2003, MNRAS, 343, 107

Chary, R., \& Elbaz, D. 2001, ApJ, 556, 562

Devriendt, J. E. G., \& Guiderdoni, B. 2000, A\&A, 363, 851

Dole, H. 2003, in Exploiting the ISO Data Archive: Infrared Astronomy in the Internet Age, ed. C. Gry, S. Peschke, J. Matagne, P. Garcia-Lario, R. Lorente, \& A. Salama (ESA SP-511) (Noordwijk: ESA), 307

Dole, H., Lagache, G., \& Puget, J. L. 2003, ApJ, 585, 617

Dole, H., et al. 2001, A\&A, 372, 364 2004, ApJS, 154, 93

Efstathiou, A., et al. 2000, MNRAS, 319, 1169

Egami, E., et al. 2004, ApJS, 154, 130

Elbaz, D. 2002, A\&A, 384, 848

Elbaz, D., et al. 1999, A\&A, 351, L37

Genzel, R., \& Cesarsky, C. J. 2000, ARA\&A, 38, 761

Gispert, R., Lagache, G., \& Puget, J. L. 2000, A\&A, 360, 1

Gordon, K. D., et al. 2004, PASP, submitted

Hacking, P. B., \& Soifer, B. T. 1991, ApJ, 367, L49

Hauser, M. G., \& Dwek, E. 2001, ARA\&A, 37, 249

Hauser, M. G., et al. 1998, ApJ, 508, 25

Helou, G., \& Beichman, C. A. 1990, in From Ground-Based to Space-Borne Sub-mm Astronomy (ESA SP-314) (Noordwijk: ESA), 117

Jannuzi, B. T., \& Dey, A. In Weymann, R. 1999, in ASP Conf. Ser. 191, Photometric Redshifts and High Redshift Galaxies, ed. R. J. Weymann, L. J. Storrie-Lombardi, M. Sawicki, \& R. J. Brunner (San Francisco: ASP), 111 Juvela, M., Mattila, K., \& Lemke, D. 2000, A\&A, 360, 813

Kawara, K., et al. 1998, A\&A, 336, L9

\section{REFERENCES}

Kawara, K., et al. 2004, A\&A, 413, 843

King, A. J., \& Rowan-Robinson, M. 2003, MNRAS, 339, 260

Krause, O. 2003, Ph.D. thesis, Ruperto-Carola Univ. Heidelberg

Lagache, G., Dole, H., \& Puget, J. L. 2003, MNRAS, 338, L555

Lagache, G., Haffner, L. M., Reynolds, R. J., \& Tufte, S. L. 2000, A\&A, 354,247

Lagache, G., et al. 1999, A\&A, 344, 322 2004, ApJS, 154, 112

Le Floc'h, E., et al. 2004, ApJS, 154, 170

Linden-Vornle, M. J. D., et al. 2000, A\&A, 359, L51

Lonsdale, C. J., Hacking, P. B., Conrow, T. P., \& Rowan-Robinson, M. 1990, ApJ, 358, L60

Matsuhara, H., et al. 2000, A\&A, 361, 407

Mould, J. 2003, ApJ, 587, L93

Papovich, C., et al. 2004, ApJS, 154, 70

Patris, J., Dennefeld, M., Lagache, G., \& Dole, H. 2003, A\&A, 412, 349

Puget, J. L., et al. 1996, A\&A, 308, L5 1999, A\&A, 345, 29

Rieke, G. H., et al. 2004, ApJS, 154, 25

Rodighiero, G., et al. 2003, MNRAS, 343, 1155

Rowan-Robinson, M., et al. 1986, MNRAS, 219, 273

Soifer, B. T., \& Neugebauer, G. 1991, AJ, 101, 354

Stetson, P. B. 1987, PASP, 99, 191

Stickel, M., et al. 2004, A\&A, in press

Werner, M., et al. 2004, ApJS, 154, 1 


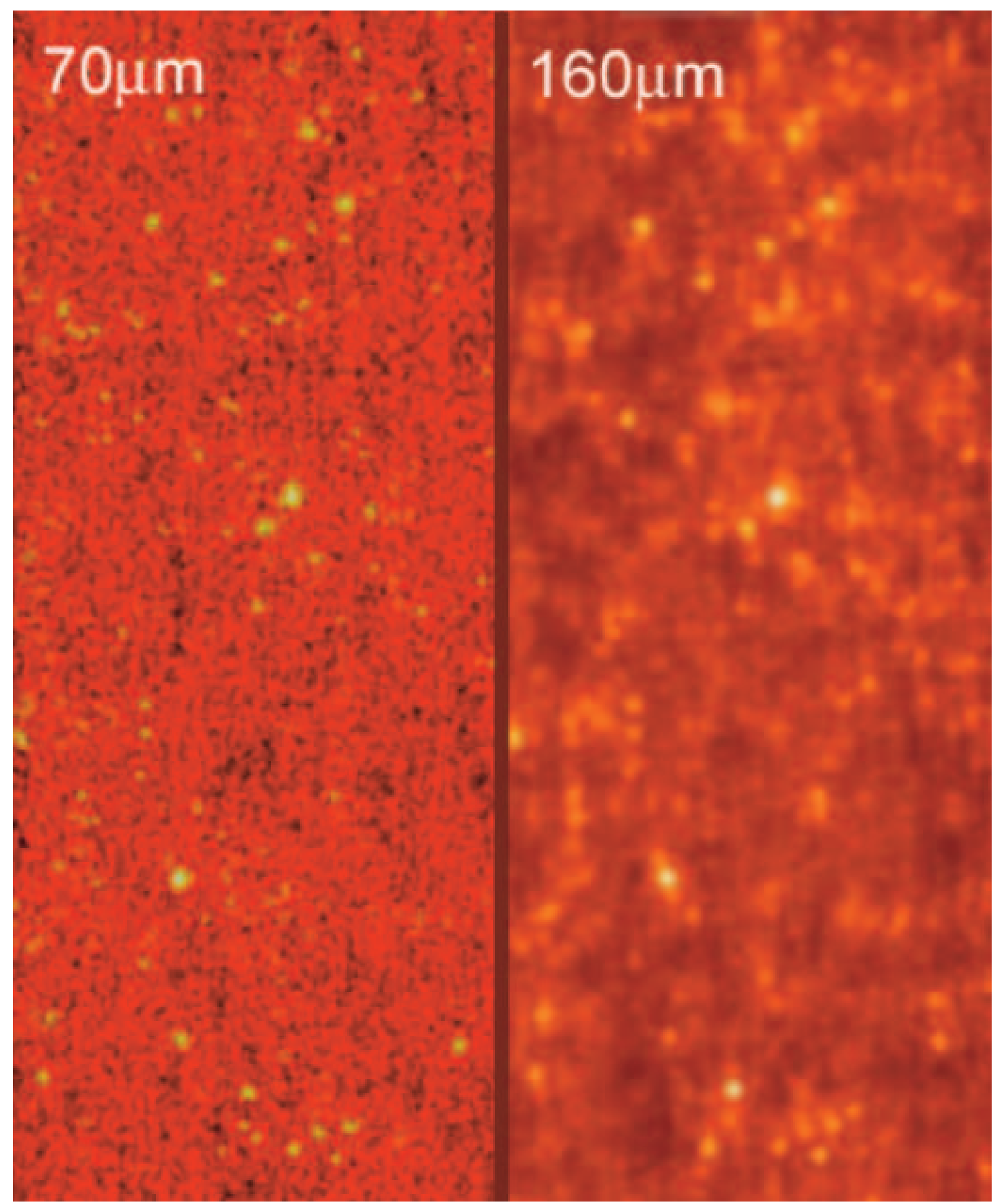

FIG. 1.-MIPS observations of the Chandra Deep Field-South at 70 and $160 \mu \mathrm{m}$. The field covers an area of $25^{\prime} \times 1^{\circ}$. 